\title{
AVALIAÇÃO FINANCEIRA DO SORGO FORRAGEIRO NO SEMIÁRIDO CEARENSE
}

\author{
Paulo Gleisson Rodrigues de Sousa* \\ Thales Vinícius de Araújo Viana** \\ Clayton Moura de Carvalho*** \\ Kilmer Coelho Campos***** \\ Francisco Limeira da Silva****** \\ Benito Moreira de Azevedo******
}

RESUMO: Objetivou-se avaliar financeiramente indicadores de viabilidade da implantação do sorgo forrageiro (BRS Ponta Negra) sob diferentes lâminas de irrigação $\left(50 ; 75 ; 100 ; 125\right.$ e 150\% da evapotranspiração da cultura, ET $\left.{ }_{C}\right)$ associadas a diferentes níveis de cobertura morta com bagana de carnaúba $(0,00 ; 2,50 ; 3,75$; $5,00$ e $6,25 \mathrm{~cm})$. O trabalho foi conduzido no Instituto Federal de Educação, Ciência e Tecnologia do Ceará (IFCE) - campus de Umirim (CE), no período de setembro de 2016 a janeiro de 2017. O delineamento experimental foi disposto em parcelas subdivididas, 5 x 5 com 5 repetições, totalizando 125 unidades experimentais. Os indicadores calculados foram: Valor presente líquido (VPL), Relação benefício-custo $(\mathrm{RB} / \mathrm{C})$, Taxa interna de retorno (TIR) e payback descontado (PB) para um horizonte de planejamento de dez anos. Pelos resultados obtidos foi possível concluir que a produção de sorgo nas condições de cultivo foi financeiramente viável para todos os indicadores em análise em todas as lâminas de irrigação aplicadas e níveis de cobertura morta, apontando essa como uma cultura promissora para a região.

PALAVRAS-CHAVE: Eficiência econômica; Manejo da irrigação; Sorghum bicolor L.

\footnotetext{
"Mestrando em Engenharia Agrícola pela Universidade Federal do Ceará - UFC, Fortaleza, Brasil. E-mail: paulo.ufc.agro@gmail.com

** Docente de Engenharia Agrícola pela Universidade Federal do Ceará - UFC, Fortaleza, Brasil.

**** Docente de Engenharia Agrícola pelo Instituto Federal de Educação, Ciência e Tecnologia Baiano - IF Baiano, Xique-Xique, BA, Brasil.

***** Docente de Economia Agrícola pela Universidade Federal do Ceará - UFC, Fortaleza, Brasil.

****** Docente de Engenharia Agrícola pelo Instituto Federal de Educação, Ciência e Tecnologia do Ceará - IFCE, Umirim, CE, Brasil.

******* Docente de Engenharia Agrícola pela Universidade Federal do Ceará - UFC, Fortaleza, Brasil.
} 


\title{
FINANCIAL EVALUATION OF FORAGE SORGHUM IN THE SEMI-ARID REGION OF THE CEARÁ, BRAZIL
}

\begin{abstract}
The viability index of forage sorghum (BRS Ponta Negra) implantation is evaluated at different irrigation laminas $(50 ; 75 ; 100 ; 125 ; 150 \%$ of evapotranspiration of culture, ETC), associated to different levels of the dead coverage with carnauba palm straw $(0.00 ; 2.50 ; 3.75 ; 5.00 ; 6.25 \mathrm{~cm})$. Research was undertaken at the Instituto Federal de Educação, Ciência e Tecnologia do Ceará (IFCE), campus Umirim (CE), between September 2016 and January 2017. Experimental design comprised subdivided plots, $5 \times 5$, with 5 replications, totaling 125 experimental units. Indexes calculated were net value (VPL), cost-benefit relationship $(\mathrm{RB} / \mathrm{C})$, Internal return rate (TIR) and discount payback (PB) for a 10-year planning. Results showed that sorghum production in culture condition was financially viable for all indicators in all irrigation laminas and levels of dead coverage, confirming a promising culture in the region.
\end{abstract}

KEY WORDS: Economic efficiency; Irrigation management; Sorghum bicolor L.

\section{INTRODUÇÃO}

O sorgo (Sorghum bicolor L.) se destaca pela alta produção de biomassa, resistência a doenças e pela grande tolerância ao déficit hídrico, sendo uma das espécies forrageiras mais versáteis e eficientes na produção de alimentares. Nas últimas décadas, essa cultura tem sido utilizada no processo de ensilagem de forma crescente, principalmente por seu fácil cultivo, pelo alto rendimento de biomassa, pela tolerância ao déficit hídrico, pelo bom desenvolvimento do sistema radicular, pela possibilidade de se cultivar a rebrota quando submetido a manejo adequado e, especialmente, pela qualidade da silagem produzida sem a necessidade de aditivo para se estimular a fermentação (XIN; AIKEN; BURKE, 2009; ALBUQUERQUE; MENDES, 2011).

Dessa forma tem-se observado que as pesquisas na agricultura têm enfocado na obtenção de máximas produtividades. No entanto, investigações realizadas por esses autores (GEERTS; RAES, 2009; KLOCKE et al., 2010) têm apontado limitações nos recursos terra e água, razão pela qual as estratégias de irrigação deficitária têm 
sido indicadas para regiões com baixo índice pluviométrico associado à distribuição irregular de chuvas.

Segundo English, Solomon e Hoffman (2002), o manejo da irrigação em condições de reduzida disponibilidade hídrica precisa resultar no melhor retorno econômico por unidade de água aplicada. A irrigação deficitária pode ser utilizada na agricultura irrigada ocasionando um mínimo de perdas de produtividade, e assim atingindo patamares elevados na eficiência de uso da água que pode ser alcançada quando a cultura é submetida ao déficit hídrico, mantendo a viabilidade econômica da produção (GEERTS; RAES, 2009; DU et al., 2010; PEREIRA; CORDERY; IACOVIDES, 2012).

Lima Júnior et al. (2011) afirmam que em decorrência do sistema de irrigação apresentar elevados custos de implantação e até mesmo de operação, muitas vezes o máximo rendimento da cultura não corresponde à maior receita líquida e a lâmina de irrigação deve ser recomendada a fim de proporcionar o maior rendimento líquido.

As decisões de negócios não são tomadas sem que tenham em vista os objetivos específicos do projeto. Para a análise de qualquer projeto se faz necessária uma abordagem de viabilidade financeira, sendo de fundamental importância o entendimento dos fluxos de caixa, ou seja, análise do valor do dinheiro no tempo, que é baseada na ideia de que uma unidade monetária hoje vale mais do que uma outra que será recebida em uma data futura (BRIGHAM; HOUSTON, 1999; GITMAN, 2001; MACEDO; LUNGA; ALMEIDA, 2007).

No momento da tomada de decisões em um determinado projeto, faz-se necessário a adoção de critérios "índices" para o entendimento das implicações financeiras do mesmo, e esse pode ser avaliado, analisando a relação benefício/ custo $(\mathrm{B} / \mathrm{C})$, taxa interna de retorno (TIR), valor presente líquido (TIR) e período "payback". Os métodos de análises do VPL e TIR estão sendo muito utilizados no setor agrícola, abrangendo diversos estudos de viabilidade financeira (ARAÚJO et al., 2009; VASCONCELOS et al., 2010).

Assim, o presente artigo objetiva avaliar financeiramente indicadores de viabilidade de implantação do sorgo forrageiro (BRS Ponta Negra) sob diferentes lâminas de irrigação $(50 ; 75 ; 100 ; 125$ e 150\% da evapotranspiração da cultura, 
ET $_{\mathrm{C}}$ ) associadas a diferentes níveis de cobertura morta com bagana de carnaúba $(0,00 ; 2,50 ; 3,75 ; 5,00$ e $6,25 \mathrm{~cm})$. Tem-se como objetivos específicos elaborar a demonstração do fluxo de caixa para um horizonte de planejamento de 10 anos e, em seguida, calcular e analisar alguns indicadores financeiros, a exemplo do valor presente líquido, relação benefício-custo e taxa interna de retorno.

\subsection{REFERENCIAL TEÓRICO}

A análise de investimentos é importante no setor agrícola, pois identifica onde, quando e como aplicar os recursos, principalmente para acompanhar um mercado competitivo e dinâmico, onde ocorrem rápidas alterações. Desta forma, considera-se conveniente medir o impacto financeiro de tecnologias, utilizando metodologias modernas e específicas para tal fim.

Os modelos de análise de investimentos são modelos matemáticos que tentam simular como as principais variáveis irão se comportar no caso dos investimentos realizados. Um modelo de análise de investimento não se propõe a prever exatamente como as variáveis vão se comportar no futuro, mas deve oferecer informações objetivas e úteis para identificar pontos fracos que possam comprometer o projeto de investimento (GALICIA, 2010).

Neste estudo, a análise está focada em duas etapas da elaboração de um projeto: construção e análise dos fluxos financeiros. Os fluxos de caixa representam as estimativas de entradas (receitas) e saídas (custos) de recursos monetários em um determinado projeto produtivo ao longo do tempo. Para Assef (2003), o fluxo de caixa mensura as necessidades futuras de recursos, a possibilidade de cumprir pontualmente com seus compromissos e a disponibilidade de recursos financeiros para investimentos.

Os principais indicadores de viabilidade de projetos destacados na literatura, e posteriormente neste trabalho, são: Valor Presente Líquido (VPL), Relação Benefício/Custo (B/C), Taxa Interna de Retorno (TIR) e payback descontado.

O VPL significa que ao se efetuar uma inversão espera-se receber, ao longo da vida desta, um valor igual à soma investida mais um valor adicional (resíduo). Essas somas que seriam recebidas são aplicadas no projeto ou inversão ao longo de 
sua vida. O VPL indica o valor resultante ao subtrair (descontar) a inversão e a soma que o investidor já recebia por sua inversão (CAMPOS; CAMPOS, 2015).

O cálculo do VPL apresenta-se como medida de análise superior à relação $\mathrm{B} / \mathrm{C}$ e a TIR, apesar destes dois últimos critérios serem extensamente usados em tomada de decisões de investir pelos empresários.

Fica evidente que o VPL e, por fim, a decisão de aceitar ou não um projeto de inversão dependem do custo de oportunidade do dinheiro. O VPL é um indicador confiável para a comparação e ordenamento de projetos porque se ampara em suposições consistentes e confiáveis: todos os fundos que não são investidos (capital excedente que excede a inversão inicial) e os fundos liberados pelo projeto ou benefício líquido gerado (os ganhos ou excedentes líquidos que se convertem em fundos que se podem reinvestir) são investidos pela taxa de juros de oportunidade.

A Relação Benefício/Custo é o quociente entre o valor presente das receitas (benefícios) a serem obtidos e o valor presente dos custos (inclusive os investimentos).

Pareja (2009) define a TIR como sendo uma medida percentual da magnitude dos benefícios proporcionados pelo projeto ao investidor. A TIR indica a rentabilidade do projeto, medida por uma taxa de juros, acima da soma investida.

O Prazo de Retorno Atualizado do Investimento (Payback Atualizado) é o tempo necessário para recuperar o investimento inicial, o qual é indicado quando o fluxo de caixa passa do negativo para o positivo. É um indicador que considera alguns fatores, como o risco e a liquidez do investimento, gerando bastante aceitação pelos investidores privados, os quais tendem a valorizar mais a liquidez do que a rentabilidade (SAUL, 1995).

\section{MATERIAL E MÉTODOS}

O experimento foi conduzido na fazenda Floresta, no Instituto Federal de Educação, Ciência e Tecnologia do Ceará (IFCE) - campus de Umirim, localizado no município de Umirim (CE) (341'7.96”S; 39²0’25.52”O), altitude de $76 \mathrm{~m}$, no período de setembro de 2016 a janeiro de 2017. De acordo com a classificação 
climatológica de Köppen, o clima local é BSw'h', o que corresponde ao clima semiárido, com chuvas irregulares e temperaturas elevadas. O total anual médio de chuva é de $807,1 \mathrm{~mm}$ (1978-2016). Durante a realização da pesquisa não foi registrada precipitação pluviométrica.

A evapotranspiração de referência (ETo) foi calculada através do método de Penman-Monteih/FAO (1991), utilizando-se o software Cropwat. Os dados de entrada para o cálculo de ETo foram obtidos de uma série histórica para o município de Pentecoste relativa ao período 1970-1998 (CABRAL, 2000), na seguinte sequência (janeiro; fevereiro; março; abril; maio; junho; julho; agosto; setembro; outubro; novembro; dezembro - 6,$15 ; 5,33 ; 4,14 ; 4,14 ; 4,28 ; 4,61 ; 5,21 ; 6,85 ; 7,83 ; 7,97 ; 7,77$; $7,27 \mathrm{~mm}$ respectivamente).

Já os coeficientes de cultivo (Kc) nos diversos estádios fenológicos foram obtidos por Allen et al. (1998) (Fase I; Fase II; Fase III; Fase IV - 0,40; 0,68; 1,14; $1,10)$.

Logo, o fator Kr, sugerido por Keller e Karmelli (1974) foi aplicado ao cálculo usual de consumo de água pela equação 1 :

$$
\mathrm{Kr}=\frac{\% \mathrm{AC}}{0,85}
$$

em que: $\mathrm{Kr}$ - fator de redução de molhamento, devendo-se adotar o valor menor $(\mathrm{Kr} \leq 1)$; \% AC - porcentagem de área coberta pela projeção da copa, sendo que essa foi feita pela projeção da copa da planta (sombra) ao meio-dia.

As lâminas de irrigação foram equivalentes a 50\%, 75\%, 100\%, 125\% e 150\% $\mathrm{da}\left(\mathrm{ET}_{\mathrm{C}}\right)$, o que resultou ao longo do ciclo (102 dias) nos totais de lâminas de 273,71; 410,$57 ; 547,42 ; 684,28 ; 821,14 \mathrm{~mm}$, respectivamente (Figura 1), cujos valores foram obtidos pela seguinte equação 2 :

$$
\mathrm{ETc}=\mathrm{Kc} . \mathrm{ETo}
$$

em que: ETc é a evapotranspiração da cultura, em mm dia ${ }^{-1}$; Kc é o coeficiente da cultura, adimensional, tabelado; ETo é a evapotranspiração de referência, em mm $\operatorname{dia}^{-1}$. 
O Gráfico 1 a seguir aponta os valores de evapotranspiração da cultura ETc e lâmina aplicada ao longo do ciclo. Logo assim deve-se considerar que os valores das lâminas aplicadas não estão equivalentes em percentuais a ETc, fato esse justificado por ter sido utilizado o fator de redução durante o ciclo, ou seja não foi irrigada toda a área conforme mostra a Equação 2 anteriormente.

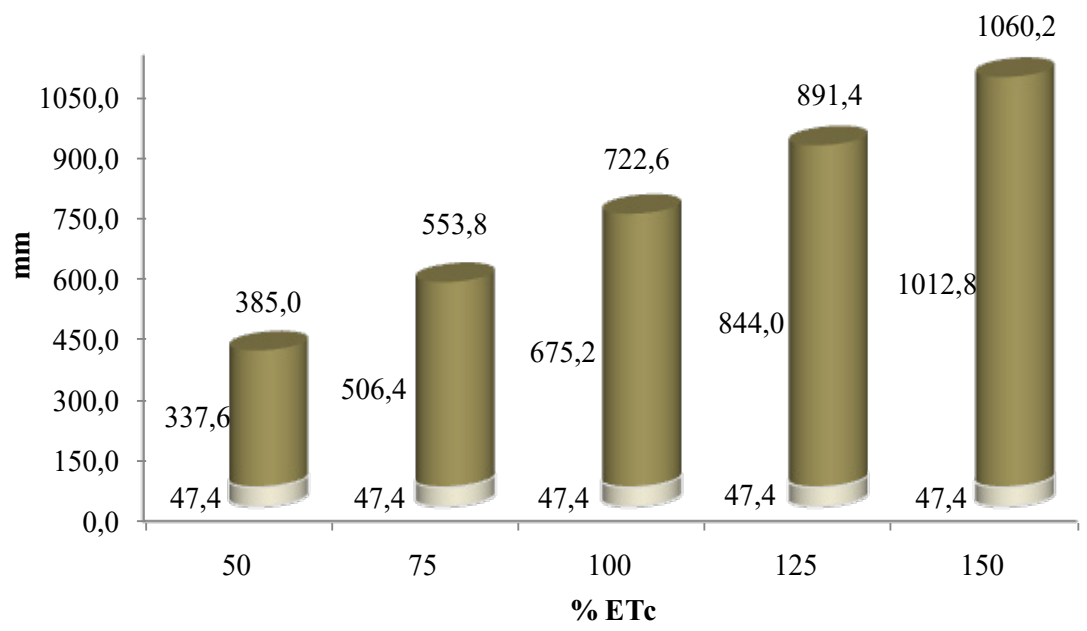

L. aplica antes da diferenciação $\square$ L. aplica após diferenciação $\quad$ L. total aplicado

Gráfico 1. Lâminas aplicadas nas fases inicial e com diferenciação e total no ciclo por tratamento

Já o fator secundário, níveis de cobertura, foi avaliado em função da resposta da altura de bagana aplicada (altura de 0,0;2,5;3,75; 5,00 e 6,25 cm, denominados $\mathrm{C}_{1}, \mathrm{C}_{2}, \mathrm{C}_{3}, \mathrm{C}_{4}$ e $\mathrm{C}_{5}$ respectivamente), sendo que essa gerou os volumes 0,$00 ; 250,00$; 375,$00 ; 500,00$ e $625,00 \mathrm{~m}^{3} \mathrm{ha}^{-1}$ respectivamente em relação a cada nível aplicado.

A área total do experimento cultivada com o sorgo foi de $1.000 \mathrm{~m}^{2}(40$ m x $25 \mathrm{~m})$. Cada parcela experimental mediu $40 \mathrm{~m}^{2}(8 \mathrm{~m} \times 5 \mathrm{~m})$, composta de 5 subparcelas de $8 \mathrm{~m}^{2}$ (1,6 m x $5 \mathrm{~m}$ ) que continham duas linhas de plantas, sendo uma de bordadura.

O delineamento experimental utilizado foi em parcela subdivida, constando de cinco tratamentos primários (lâminas de irrigação), associados a cinco tratamentos secundários (subparcelas, níveis de cobertura morta), com cinco repetições cada, totalizando 125 subparcelas. 
Foram utilizadas apenas cinco das dez fileiras como úteis para a obtenção de dados, sendo as demais consideradas como bordadura. Na fileira de plantas úteis também foram consideradas as plantas das extremidades como bordadura, ou seja, dos cinco metros de cada subparcela apenas os dois metros centrais de cada fileira foram utilizados para análise, ou seja, 1,5 $\mathrm{m}$ do início e do final foram considerados como bordadura.

Os atributos físicos e químicos do solo na camada de $(0-0,2 \mathrm{~m})$ foram determinados no Laboratório de Solo e Água do Departamento de Ciências do Solo, pertencente ao Centro de Ciências Agrárias da Universidade Federal do Ceará, sendo os atributos químicos na camada de $(0-0,2 \mathrm{~m}): \mathrm{P}\left(\mathrm{mg} \mathrm{kg}^{-1}\right) ; \mathrm{K}^{+}\left(\mathrm{cmol}_{\mathrm{c}} \mathrm{kg}^{-1}\right) ; \mathrm{Na}^{+}$ $\left(\mathrm{cmol}_{\mathrm{c}} \mathrm{kg}^{-1}\right) ; \mathrm{Ca}^{2+}\left(\mathrm{cmol}_{\mathrm{c}} \mathrm{kg}^{-1}\right) ; \mathrm{Mg}^{2+}\left(\mathrm{cmol}_{\mathrm{c}} \mathrm{kg}^{-1}\right) ; \mathrm{Al}^{3+}\left(\mathrm{cmol}_{\mathrm{c}} \mathrm{kg}^{-1}\right) ; \mathrm{M}$. O. $\left(\mathrm{g} \mathrm{kg}^{-1}\right)$; C/N; pH; CE (dS m $\left.{ }^{-1}\right)$; PST: 1,00; 0,36; 0,09; 1,80; 1,20; 0,20; 16,96; 11,00; 5,60; 0,09; 1,00, respectivamente; e os atributos físicos Areia (\%); Silte (\%); Argila (\%): 53,80; 22,40; 23,80, respectivamente.

O sistema de irrigação utilizado foi o de tipo gotejamento com emissores espaçados de $0,3 \mathrm{~m}$, operando a uma vazão de $1,6 \mathrm{~L} \mathrm{~h}^{-1}$, com pressão de $1 \mathrm{kgf} \mathrm{cm}^{-2}$. O sistema foi constituído de uma linha principal de tubos de PVC (diâmetro de 50 $\mathrm{mm}$ ), com linhas laterais constituídas por mangueiras de polietileno (diâmetro de $16 \mathrm{~mm})$.

O requerimento hídrico da cultura foi calculado para os diferentes meses e estádios, conforme equação 3 a seguir:

$$
\mathrm{Ti}=60 * \frac{\mathrm{fi} * \mathrm{ETo} * \mathrm{Kc} * \mathrm{Ap} * \mathrm{Kr}}{\mathrm{N} * \mathrm{qe}}
$$

sendo: Ti: tempo de irrigação, em minutos; fi: fator de ajuste em conformidade com os tratamentos com lâminas, 0,$50 ; 0,75 ; 1,00,1,25$ e 1,50 adimensional; ETo: evapotranspiração de referência diária, mm; Kc: coeficiente de cultivo da cultura, adimensional; Ap: área útil por planta, $\mathrm{m}^{2}$; $\mathrm{Kr}$ : coeficiente de redução, em percentagem, adimensional; $\mathrm{N}$ : número de emissores por planta, adimensional; qe: vazão do emissor, $\mathrm{L} \mathrm{h}^{-1}$.

Antes do estabelecimento da cultura do sorgo no campo foi verificada a 
necessidade de calagem para a correção do pH do solo, tomando como referência o manual de recomendação de adubação e calagem para o Estado de Pernambuco, segundo aproximações. As adubações de cobertura foram realizadas via fertirrigação com os seguintes adubos: ureia, mono-amônio fosfato (MAP) e cloreto de potássio branco.

Utilizou-se na análise de investimentos o cálculo de indicadores financeiros tais como o valor presente líquido (VPL), relação benefício-custo $(\mathrm{RB} / \mathrm{C})$, taxa interna de retorno (TIR) e período payback (PB) para um horizonte de dez anos de planejamento, de acordo com as políticas de financiamento do banco investidor. Os indicadores foram calculados por meio do programa TirART_v.2012.

Para o valor presente líquido (VPL) calculado, pode-se afirmar que é a transferência para o instante atual de todas as variações de caixa esperado, descontados a uma determinada taxa de juros, e somados algebricamente, sendo representado pelo valor presente dos Benefícios Líquidos (Benefícios-Custos), conforme Equação 4 (HOFFMANN et al., 1981).

$$
\mathrm{VPL}=\frac{(R i-C i)}{(1+r)^{i}}=\sum_{i=0}^{n} \frac{R i}{(1+r)^{i}}-\sum_{i=0}^{n} \frac{C i}{(1+r)^{i}}
$$

em que: Ri: Receita líquida obtida no ano em $(\mathrm{R} \$)$; r: Taxa real anual de juros (decimal); i: Número 0, 1, 2,...,n (anos); Ci: Custos no ano.

A relação benefício-custo $(\mathrm{B} / \mathrm{C})$ representa o valor presente das receitas obtidas e o valor presente dos custos (inclusive os investimentos). O critério de decisão é que o investimento será considerado viável se a relação benefício/ custo $(\mathrm{B} / \mathrm{C})>1$. Para o experimento, a relação $\mathrm{B} / \mathrm{C}$ foi calculada pela Equação 5 (HOFFMANN et al., 1981):

$$
\frac{B}{C}=\frac{\frac{\sum_{i=0}^{n}(R i-C i)}{(1+r)^{i}}}{\frac{\sum_{i=0}^{n} C i}{(1+r)^{i}}}
$$


em que: Ri: Receita bruta obtida no ano em (R\$); Ci: Custos anuais da produção (R\$); r: Taxa real anual de juros (decimal); i: Número de $0,1,2, \ldots, n$ (anos); Ci: Custos no ano.

A taxa interna de retorno (TIR) é uma variável que expressa a percentagem de rentabilidade anual do capital alocado durante todo o horizonte de análise, sendo, portanto, uma taxa que torna nula o VPL do fluxo de caixa de investimento, caracterizando, assim, a taxa de remuneração do capital investido, conforme Equação 6 (HOFFMANN et al., 1981):

$$
T I R=\mathrm{r} * \quad \text { tal que } \sum_{i=0}^{n} \frac{(\mathrm{Ri}-\mathrm{Ci})}{(1+\mathrm{r} *)^{\mathrm{i}}}=0
$$

em que: Ri: Receita bruta obtida no ano em (R\$); Ci: Custos anuais da produção; r*: Taxa real anual de juros que anula o PVL (decimal); i: Número 0, 1, $2, \ldots, \mathrm{n}$ (anos); Ii: Investimento inicial do projeto.

O período payback, ou simplesmente Payback, é o tempo necessário para que se tenha o retorno do capital investimento em um projeto (HOFFMANN et al., 1981). Assim sendo no presente projeto foi utilizado payback descontado, o que considera o tempo de recuperação do capital investido, remunerado a uma taxa mínima de atratividade, de modo que essa foi considerada para receitas e custos normais a taxa de desconto do projeto de investimento de $2,5 \%$ a. a., conforme Equação 7.

$$
P B=\sum_{i=0}^{n} \frac{\mathrm{Ri}}{(1+\mathrm{r})^{\mathrm{i}}}-I i=0
$$

em que: Ri: Receita líquida obtida no ano em (R\$); r: Taxa real anual de juros (decimal); i: Número 0, 1, 2,...,n (anos); Ii: Investimento inicial do projeto.

$\mathrm{Na}$ análise dos investimentos, foi considerado que o agricultor obteve financiamento junto ao Banco do Nordeste do Brasil (BNB), se enquadrando na 
linha de credito "Pronaf Semiárido" para agricultores familiares enquadrados nos Grupos A, A/C, B e Grupo V (renda variável) que tem como finalidade propiciar crédito para investimento destinado à convivência com o semiárido, priorizando a infraestrutura hídrica. Desse modo, essa linha de crédito apresenta uma forma de financiamento com prazo de até dez anos para o pagamento e carência de até três anos, a uma taxa de juros de 2,5\% ao ano (BANCO DO NORDESTE DO BRASIL, 2016).

O critério de decisão para o referido horizonte do projeto de "dez anos" se constitui no tempo de pagamento do financiamento, tendo em vista que esse se caracteriza como um valor relativamente baixo e os materiais utilizados não influenciaram nessa decisão.

Os preços de todos os insumos e produtos utilizados na análise financeira foram coletados na região de realização da pesquisa, "Vale do Curu", para se identificar o potencial econômico das alternativas testadas.

Desse modo, os custos foram obtidos com base nos itens a seguir, de maneira que os preços dos insumos e equipamentos foram levantados em lojas agropecuárias de Maracanaú (CE), e na região de desenvolvimento da pesquisa em junho de 2016:

- Pode-se destacar como custo inicial à aquisição do material de irrigação, ferramentas (enxada, foice, matraca etc.), cercamento do terreno;

- Para as operações manuais foi considerada a contratação de um operário, em que o custo com a contratação foi considerado o salário acrescido dos direitos trabalhistas, somado a diárias para as operações de plantio e colheita;

- Os gastos com insumos e materiais foram obtidos pelo produto entre a quantidade dos materiais usados e os seus respectivos preços de mercado;

- O custo com horas máquina foi obtido pelo produto entre o número de horas necessário para todas as operações e o preço da mesma no mercado; 
- Para a manutenção dos investimentos, foi considerada uma taxa de 3\% ao ano sob o valor do investimento;

- Foi considerado o arrendamento da terra de $\mathrm{R} \$ 1.200,00$ como sendo um custo de oportunidade para esse bem;

- O custo da água foi calculado pelo produto entre o volume $\mathrm{em}^{-3} \mathrm{de}$ água usado durante o ano e o valor do mesmo, sendo considerado um custo de oportunidade mínimo de $\mathrm{R} \$ 0,15$;

- Para o custo com assistência técnica, encargo com associação e Funrural foi considerada uma taxa de $6 \%$ sob $80 \%$ da receita bruta obtida no ano;

- O valor da tarifa de energia elétrica foi formado pela soma do custo do consumo efetivo da energia e do custo de demanda da potência elétrica. Pelas normas da ANEEL (Agência Nacional de Energia Elétrica), só existe tarifa de demanda quando a potência instalada é superior a $75 \mathrm{KVA}$. Logo, para as condições da pesquisa o sistema operou com uma potência instalada bem inferior, com um motor elétrico de $4,0 \mathrm{cv}$ para irrigar um hectare, sendo que o custo de demanda foi nulo, e a tarifa de energia composta apenas pelo custo do consumo, conforme mostrado na Equação 8 (FRIZZONE; BOTREL; FREITAS, 1994):

\section{$\mathrm{CE}=0,7457 *$ Pot $*$ Tf $*$ Pkwh}

sendo: CE: custo da energia elétrica durante o ciclo da cultura, em R\$; 0,7457: fator de conversão de cv para kw; Pot: potência do motor, em cv; Tf: tempo de funcionamento do sistema necessário, em horas, durante um ano e considerando uma área irrigada de 1,0 ha que variou com os tratamentos em função dos percentuais da ETc; Pkwh: preço do kwh, em R\$.

A receita bruta $(\mathrm{RB})$ em $(\mathrm{R} \$)$ foi obtida pelo produto entre a produtividade em tonelada de silagem de sorgo e a média dos preços praticados para esse produto entre abril a dezembro de 2016.

O custo de aquisição da bagana para cobertura morta foi constituído pelo 
produto entre o preço praticado $\mathrm{R} \$ 62,50$ por $\mathrm{m}^{-3} \mathrm{e}$ o volume utilizado nos diferentes tratamentos.

Dessa forma, para fins de análise financeira, cada tratamento foi considerado como uma lavoura comercial com uma área de um hectare, em que todos os insumos foram considerados iguais, variando apenas os custos na aplicação das diferentes lâminas aplicadas e os diferentes níveis de cobertura morta com bagana de carnaúba. Nos custos operacionais foi considerada uma variação no número de horas de máquinas para colheita, com base na estimativa de biomassa colhida por hora de serviço.

\section{RESULTADOS E DISCUSSÃO}

Os indicadores de viabilidade financeira estão organizados conforme os fatores de diferentes lâminas de irrigação e níveis de cobertura morta.

$\mathrm{Na}$ Tabela 1 são apresentados os resultados dos indicadores de projeto: relação benefício/custo $(\mathrm{B} / \mathrm{C})$, valor presente líquido (VPL), taxa interna de retorno (TIR) e o período payback (PB) calculados para um período de fluxo de caixa de dez anos, em função das lâminas de irrigação aplicadas na cultura do sorgo.

Tabela 1. Indicadores financeiros: (B/C), (VPL), (TIR) e (PB) em função da lâmina de irrigação aplicada na cultura do sorgo com obtenção de financiamento, Umirim, Ceará, 2016

\begin{tabular}{ccccc}
\hline & \multicolumn{4}{c}{ Indicadores } \\
\cline { 2 - 5 } & VPL $(\mathrm{R} \$)$ & $\mathrm{B} / \mathrm{C}$ & TIR $(\%)$ & PB (anos) \\
\hline $\mathrm{L}_{1}$ & $13.921,05$ & 1,04 & 29,28 & 9,59 \\
$\mathrm{~L}_{2}$ & $42.340,49$ & 1,11 & 124,59 & 4,14 \\
$\mathrm{~L}_{3}$ & $36.796,41$ & 1,09 & 97,45 & 4,51 \\
$\mathrm{~L}_{4}$ & $90.045,94$ & 1,23 & 30,78 & 2,19 \\
$\mathrm{~L}_{5}$ & $99.031,33$ & 1,24 & 31,12 & 2,03 \\
\hline
\end{tabular}

Pelos valores expressos na Tabela 1, verifica-se que todos os tratamentos mostraram-se viáveis em termos financeiros, com valores de VPL positivo, altas taxas de retorno (TIR), sendo essas sempre maior que um, e períodos PB relativamente curtos, exceto para a lâmina $\mathrm{L}_{1}$ com um tempo de retorno do capital investido em 
9,59 anos. Os melhores indicadores econômicos foram obtidos para o tratamento em que a irrigação foi equivalente a $150 \%$ da ETc, com VPL de R\$ 99.031,33; TIR de 31,12\%; B/C 1,24; e PB de 2,03 anos, tempo necessário ao produtor recuperar o capital investido.

Porém, analisando o comportamento da menor lâmina aplicada, $\mathrm{L}_{1}$, constatou-se que a mesma apresentou a menor viabilidade diante das demais, pois o VPL de $\mathrm{R} \$ 13.921,05$ e $\mathrm{RB} / \mathrm{C}$ de 1,04 implicam em baixa viabilidade, o que se confirma quando verifica o PB, sendo esse de 9,59 anos, o que está aproximado ao horizonte de análise do projeto.

Desse modo pode-se perceber pelos resultados que o incremento nas lâminas aplicadas gerou um aumento na viabilidade do projeto, principalmente verificando as principais variáveis de tomada de decisão que são o VPL e RB/C. Tais resultados estão diretamente influenciados com as produtividades obtidas nas diferentes lâminas, $\theta$ que decidem os valores de receita bruta.

Logo, tem que ser considerados principalmente os fatores limitantes à produção, quando se consideram água e terra como os principais, deve-se analisar qual a situação que melhor responde economicamente ao produtor. Mesmo em escalas menores, todos os tratamentos como já mencionado anteriormente foram altamente viáveis, o que pode se levar em consideração no momento da tomada de decisão, pois o volume de água utilizado na lâmina com $50 \%$ da ETc é três vezes menor que o tratamento com $150 \%$ da ETc.

As simulações de receita líquida obtida com os diferentes níveis de irrigação deficitária na cultura do sorgo realizada por Zwirtes et al., 2015 apresentaram um comportamento quadrático com as lâminas aplicadas de 25 a 100\% da ETc, em que a máxima eficiência econômica observada foi de $\mathrm{R} \$ 1.375,00$ com a aplicação de irrigação deficitária de 75\% da ETc.

Martin et al. (2012) avaliaram a silagem de plantas de milho submetidas aos manejos de irrigação plena (100\% ETc), de irrigação com déficit hídrico leve ( $75 \%$ ETc) e moderado ( $50 \%$ ETc), os autores verificaram que o retorno econômico alcançado com as irrigações deficitárias na produção de silagem de milho foi maior que o observado com a irrigação plena.

Foi realizada uma análise considerando uma variação nas taxas de desconto sob o capital de investimento, no sentido de se investigar mais um indicador para a 
tomada de decisão do projeto, pois o capital investido pode ser mais atrativo para outras fontes de investimento, como, por exemplo, uma poupança.

De acordo com os resultados apresentados na Tabela 2, pode-se verificar que o capital aplicado na atividade de produção de sorgo em um hectare, nas diferentes condições de cultivo, apresentou viabilidade financeira, quando os fluxos foram atualizados às taxas de desconto de $4 \%$ a $12 \%$ a. a.

O melhor indicador para essa análise foi observado na condição uma taxa de desconto de $4 \%$ a. a. A relação benefício-custo mostrou-se maior que um para todos os tratamentos, em todas as taxas aplicadas, com destaque para a lâmina $\mathrm{L}_{5}$, correspondente 1,24 , indicando que os benefícios gerados a partir das receitas obtidas superam os custos e para cada $\mathrm{R} \$ 1,00$ gasto no projeto tem-se um retorno bruto de $\mathrm{R} \$ 1,24$ e um retorno líquido de $\mathrm{R} \$ 0,24$.

Tabela 2. Avaliação financeira da produção de sorgo em função da lâmina de irrigação aplicada, Umirim, Ceará, 2016

\begin{tabular}{|c|c|c|c|c|c|c|}
\hline & \multicolumn{2}{|c|}{$\mathrm{L}_{1}$} & \multicolumn{2}{|c|}{$\mathrm{L}_{2}$} & \multicolumn{2}{|c|}{$\mathrm{L}_{3}$} \\
\hline & VPL & $\mathrm{RB} / \mathrm{C}$ & VPL & $\mathrm{RB} / \mathrm{C}$ & VPL & $\mathrm{RB} / \mathrm{C}$ \\
\hline $4 \%$ & $12.131,38$ & 1,03 & $38.468,84$ & 1,11 & $33.330,91$ & 1,09 \\
\hline $6 \%$ & $10.080,64$ & 1,03 & $33.980,09$ & 1,10 & $29.317,77$ & 1,09 \\
\hline $8 \%$ & $8.349,66$ & 1,03 & $30.138,44$ & 1,10 & $25.887,87$ & 1,08 \\
\hline $10 \%$ & $6.882,03$ & 1,03 & $26.834,49$ & 1,10 & $22.942,15$ & 1,08 \\
\hline \multirow[t]{3}{*}{$12 \%$} & $5.632,36$ & 1,02 & $23.979,60$ & 1,09 & $20.400,41$ & 1,08 \\
\hline & \multicolumn{2}{|c|}{$\mathrm{L}_{4}$} & \multicolumn{4}{|c|}{$\mathrm{L}_{5}$} \\
\hline & VPL & $\mathrm{RB} / \mathrm{C}$ & \multicolumn{2}{|c|}{ VPL } & \multicolumn{2}{|c|}{$\mathrm{RB} / \mathrm{C}$} \\
\hline $4 \%$ & $82.326,48$ & 1,22 & \multicolumn{2}{|c|}{$90.653,61$} & \multicolumn{2}{|c|}{1,24} \\
\hline $6 \%$ & $73.379,55$ & 1,22 & \multicolumn{2}{|c|}{$80.935,86$} & \multicolumn{2}{|c|}{1,23} \\
\hline $8 \%$ & $65.723,74$ & 1,21 & \multicolumn{2}{|c|}{$72.612,72$} & \multicolumn{2}{|c|}{1,23} \\
\hline $10 \%$ & $59.139,17$ & 1,21 & \multicolumn{2}{|c|}{$65.447,55$} & \multicolumn{2}{|c|}{1,22} \\
\hline $12 \%$ & $53.447,94$ & 1,20 & \multicolumn{2}{|c|}{$59.248,80$} & \multicolumn{2}{|c|}{1,22} \\
\hline
\end{tabular}

O VPL para todos os tratamentos também mostrou viabilidade, segundo a tendência da $\mathrm{RB} / \mathrm{C}$ com o melhor indicador para a lâmina $\mathrm{L}_{5}$, sendo essa

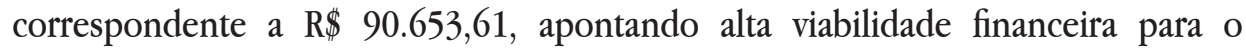
investidor privado, tendo em vista este valor indicar que o capital investido está 
sendo recuperado, pagando os custos operacionais e remunerando o capital a custo de oportunidade de $4 \%$ a. a.

Os resultados apresentados na Tabela 3 são referentes aos indicadores: valor presente líquido (VPL), relação benefício/custo (RB/C), taxa interna de retorno (TIR) e o período payback ( $\mathrm{PB}$ ) calculados para um período de fluxo de caixa de dez anos, em função dos níveis de cobertura morta aplicada na cultura do sorgo.

Tabela 3. Indicadores financeiros: (RB/C), (VPL), (TIR) e (PB) em função dos níveis de cobertura morta aplicados na cultura do sorgo com obtenção de financiamento, Umirim, Ceará, 2016

\begin{tabular}{ccccc}
\hline & \multicolumn{4}{c}{ Indicadores } \\
\cline { 2 - 5 } & VPL $(\mathrm{R} \$)$ & $\mathrm{B} / \mathrm{C}$ & TIR $\%$ & PB anos \\
\hline $\mathrm{C}_{1}$ & $88.767,05$ & 1,28 & 35,54 & 0,88 \\
$\mathrm{C}_{2}$ & $79.462,11$ & 1,22 & 31,70 & 2,10 \\
$\mathrm{C}_{3}$ & $41.040,27$ & 1,10 & 78,87 & 4,71 \\
$\mathrm{C}_{4}$ & $42.639,23$ & 1,10 & 50,25 & 5,67 \\
$\mathrm{C}_{5}$ & $55.017,29$ & 1,12 & 50,38 & 4,73 \\
\hline
\end{tabular}

Pelos valores expressos na Tabela 3, todos os tratamentos apresentaram alta viabilidade econômica financeira, sendo que o tratamento mais promissor foi o que não se utilizou cobertura morta, apresentando um VPL positivo de R $\$ 88.767,05$, indicando alta viabilidade, por apresentar uma condição de pagamento de todo o capital investido mais os custos operacionais e uma taxa de custo de oportunidade sob investimento considerada no projeto, sendo essa de $2,5 \%$ a. a. ao longo do horizonte do projeto, onde o mesmo foi de 10 anos gerando assim ao final um valor líquido de $R \$ 88.767,05$. Para a $R B / C$ de 1,28 , implicando que para cada $R \$ 1$ gasto no projeto, retorna $\mathrm{R} \$ 1,28$ bruto e $\mathrm{R} \$ 0,28$ líquido. Há de se destacar nessa análise que o PB apresentado de 0,88 anos sinaliza um alto retorno do capital, pois é considerado muito curto para o horizonte do projeto.

Analisando os demais tratamentos, onde se inseriu diferentes níveis de cobertura morta, a viabilidade do projeto foi considerada menor, porém aceitável para todos, com indicadores de viabilidade financeira bem favoráveis. Porém vale ressaltar que o aumento na quantidade de bagana aplicada traz uma redução na viabilidade do projeto. 
Com a finalidade de verificar a resistência do projeto em suportar maiores taxas existentes no mercado e assim analisar o custo de oportunidade para o capital investido foi realizada uma simulação conforme os resultados apresentados na Tabela 4, onde se pode verificar que o capital aplicado na atividade de produção de sorgo em um hectare, para os diferentes níveis de cobertura morta aplicados, apresentou viabilidade financeira, quando os fluxos foram atualizados às taxas de desconto de $4 \%$ a $12 \%$ a. a.

Tabela 4. Efeito da variação nas taxas aplicadas ao investimento sob a (B/C) e (VPL) em função dos níveis de cobertura morta na cultura do sorgo, Umirim, Ceará, 2016

\begin{tabular}{|c|c|c|c|c|c|c|}
\hline & \multicolumn{2}{|c|}{$\mathrm{C}_{1}$} & \multicolumn{2}{|c|}{$\mathrm{C}_{2}$} & \multicolumn{2}{|c|}{$\mathrm{C}_{3}$} \\
\hline & VPL & $\mathrm{RB} / \mathrm{C}$ & VPL & $\mathrm{RB} / \mathrm{C}$ & VPL & $\mathrm{RB} / \mathrm{C}$ \\
\hline $4 \%$ & $82.331,33$ & 1,28 & $72.870,29$ & 1,21 & $36.825,92$ & 1,10 \\
\hline $6 \%$ & $74.815,78$ & 1,28 & $65.221,64$ & 1,21 & $31.988,61$ & 1,10 \\
\hline $8 \%$ & $68.327,97$ & 1,28 & $58.667,87$ & 1,20 & $27.895,75$ & 1,09 \\
\hline $10 \%$ & 62. 697,98 & 1,28 & $53.023,18$ & 1,20 & $24.415,40$ & 1,09 \\
\hline \multirow[t]{3}{*}{$12 \%$} & $57.787,65$ & 1,28 & $48.137,16$ & 1,20 & $21.441,54$ & 1,08 \\
\hline & \multicolumn{2}{|c|}{$\mathrm{C}_{4}$} & \multicolumn{4}{|c|}{$\mathrm{C}_{5}$} \\
\hline & VPL & $\mathrm{RB} / \mathrm{C}$ & \multicolumn{2}{|c|}{ VPL } & \multicolumn{2}{|c|}{$\mathrm{RB} / \mathrm{C}$} \\
\hline $4 \%$ & $37.882,72$ & 1,10 & \multicolumn{2}{|c|}{$48.928,96$} & \multicolumn{2}{|c|}{1,12} \\
\hline $6 \%$ & $32.436,22$ & 1,09 & \multicolumn{2}{|c|}{$41.948,57$} & \multicolumn{2}{|c|}{1,11} \\
\hline $8 \%$ & $27.841,39$ & 1,08 & \multicolumn{2}{|c|}{$36.051,21$} & \multicolumn{2}{|c|}{1,10} \\
\hline $10 \%$ & $23.946,31$ & 1,08 & \multicolumn{2}{|c|}{$31.044,91$} & \multicolumn{2}{|c|}{1,10} \\
\hline $12 \%$ & $20.629,04$ & 1,07 & \multicolumn{2}{|c|}{$26.775,40$} & \multicolumn{2}{|c|}{1,09} \\
\hline
\end{tabular}

Pelos resultados aqui apresentados, pode-se verificar que o projeto apresentou uma boa margem de segurança para o investidor privado, tendo em vista o tratamento com menor viabilidade $\mathrm{C}_{5}$, qual corresponde ao maior volume de bagana por unidade de área, $625 \mathrm{~m}^{3} \mathrm{ha}^{-1}$, apresentou um VPL positivo de $\mathrm{R} \$$ $26.775,40$ a uma taxa de $12 \%$ a. a. e uma RB/C de 1,09, sendo o VPL superior ao rendimento de uma poupança a $7 \%$ a. a.

Os resultados para os tratamentos aos níveis de cobertura morta inferior ao $\mathrm{C}_{5}$ apresentaram maior viabilidade, em que a melhor condição foi observada para todos os tratamentos com a taxa de $4 \%$ a. a., mediante os indicadores VPL e RB/C. 


\section{CONSIDERAÇÕES FINAIS}

De acordo com os resultados obtidos pelos indicadores financeiros, a produção de sorgo nas condições de cultivo foi viável para todos os indicadores em análise em todas as lâminas de irrigação aplicadas e níveis de cobertura morta, para as condições de financiamento, apontando essa como uma cultura promissora para a região Nordeste.

Logo, as políticas de fortalecimento da agricultura familiar com acesso ao crédito e assistência técnica são de fundamental importância para a estruturação física do pequeno produtor no mercado de forma a proporcionar uma produção viável financeiramente.

O processo de avaliação de investimentos do sorgo forrageiro sob diferentes lâminas de irrigação associadas a diferentes níveis de cobertura morta com bagana de carnaúba demandou uma série de informações financeiras que são influenciadas por diferentes variáveis agronômicas e econômicas e que interferem nos critérios de análise de investimentos. Logo, caracterizar e sumarizar métodos ou ferramentas que considerem o impacto desses fatores, permitindo elucidar uma proposta de projeto em termos de lucratividade, pode proporcionar importantes benefícios.

É sugestivo testar essa técnica de produção em outras culturas e em mais sub-regiões do semiárido e assim comprovar a viabilidade das propostas de investimentos.

\section{REFERÊNCIAS}

ALBUQUERQUE, C. J. B.; MENDES, M. C. Época de semeadura do sorgo forrageiro em duas localidades do estado de Minas Gerais. Tecnologia Aplicada nas Ciências Agrárias, v. 4, n. 1, p. 116-134, jan./abr. 2011.

ALLEN, R. G.; PEREIRA, L. S.; RAES, D.; SMITH, M. Crop evapotranspiration: guidelines for computing crop water requirements. Rome: FAO, 1998. 174 p. (FAO Irrigation and Drainage Paper, 56).

ARAÚJO, A. C.; VIRGENS FILHO, A. C.; SILVA, L. M. R.; ARAÚJO, L. V. Análise da via- 
bilidade financeira da cultura da acerola no agropolo do Vale do Rio das Contas no Estado da Bahia. In: CONGRESSO BRASILEIRO DE ECONOMIA, ADMINISTRAÇÃO E SOCIOLOGIA RURAL, 47., 2009, Porto Alegre. Anais [...] Porto Alegre: SOBER, 2009.

ASSEF, R. Guia prático de administração financeira: pequenas e médias empresas. 2. ed. Rio de Janeiro: Elsevier, 2003.

BANCO DO NORDESTE DO BRASIL. Manual básico: operações de crédito. Fortaleza: BNB, 2016. Cap. 27.

BRIGHAM, E. F.; HOUSTON, J. F. Fundamentos da moderna administração financeira. Rio de Janeiro: Campus, 1999.

CABRAL, R. C. Evapotranspiração de referência de Hargreaves (1974) corrigida pelo método Penman-Monteith/FAO (1991) para o Estado do Ceará. 2000. 83 f. Dissertação (Mestrado em Irrigação e Drenagem) - Universidade Federal do Ceará, Fortaleza, 2000.

CAMPOS, R. T.; CAMPOS, K. C. Elaboração e avaliação de projetos agropecuários. Fortaleza: UFC/CCA/DEA, 2015. Notas de aula.

DU, T.; KANG, S.; SUN, J.; ZHANG, X.; ZHANG, J. An improved water use efficiency of cereals under temporal and spatial deficit irrigation in north China. Agricultural Water Management, v. 97, n. 1, p. 66-74, 2010.

ENGLISH, M. J.; SOLOMON, K. H.; HOFFMAN, G. J. A paradigm shift in irrigation management. Journal of Irrigation and Drainage Engineering, v. 128, n. 5, p. 267-277, 2002.

FRIZZONE, J. A.; BOTREL, T. A.; FREITAS, H. A. C. Análise comparativa dos custos de irrigação por pivô-central, em cultura de feijão, utilizando energia elétrica e óleo diesel. Engenharia Rural, v. 5, n. 1, p. 34-53, 1994.

GALICIA, S. A. Cómo valorar un proyecto de inversión: manuales prácticos de gestión. Santiago de Compostela, España, 2010. 
GEERTS, S.; RAES, D. Deficit irrigation as anon-farm strategy to maximize crop water productivity in dry areas. Agricultural Water Management, v. 96, n. 9, p. 1275-1284, 2009.

GITMAN, L. J. Princípios de administração financeira. 2. ed. Porto Alegre: Bookman, 2001.

HOFFMANN, R.; ENGLER, J. J. de C.; SERRANO, O.; THAME, A. C. de M.; NEVES, E. M. Administração da empresa agrícola. 3. ed. São Paulo: Pioneira, 1981. 325 p.

KELLER, J.; KARMELI, D. Trickle irrigation design. Transactions of the ASAE, v. 17, n. 4, p. 678-684, 1974.

KLOCKE, N. L.; CURRIE, R. S.; STONE, L. R.; BOLTON, D. A. Planning for deficit irrigation. Applied Engineering in Agriculture, v. 26, n. 3, p. 405-412, 2010.

LIMA JÚNIOR, J. A.; PEREIRA, G. M.; GEISENHOFF, L. O.; COSTA, G. G.; REIS, R. P.; OLIVEIRA, L. F. C. Avaliação econômica da produção de alface americana em função de lâminas de irrigação. Ciência e Agrotecnologia, v. 35, n. 2, p. 392-398, 2011.

MACEDO, M. A. da S.; LUNGA, A.; ALMEIDA, K. Análise de análise de viabilidade econômico-financeira de projetos agropecuários: o caso da implantação de um projeto de produção de produtos apícolas. In: CONGRESSO BRASILEIRO DE ECONOMIA, ADMINISTRAÇÃO E SOCIOLOGIA RURAL, 45., 2007, Londrina. Anais [...]. Londrina: SOBER, 2007.

MARTIN, J. D. et al. Irrigação deficitária para aumentar a produtividade da água na produção de silagem de milho. Irriga, ed. esp., p. 192-205, 2012.

PAREJA, I. V. Decisiones de inversión para la valoración financiera de proyectos y empresas. 6. ed. Buenos Aires: Fondo Editorial Consejo, 2009. 692 p.

PEREIRA, L. S.; CORDERY, I.; IACOVIDES, I. Improved indicators of water use performance and productivity for sustainable water conservation and saving. Agricultural Water Management, v. 108, p. 39-51, 2012. 
SAUL, N. Análise de investimentos: critérios de decisão de desempenho nas maiores empresas do Brasil. 2. ed. Porto Alegre: Ortiz, 1995. 238 p.

VASCONCELOS, L. C.; MAYORGA, F. D. de O.; TABOSA, F. J. S.; OLIVEIRA, S. C. de; PARENTE, T. D. Análise de viabilidade econômica dos pequenos produtores de banana da associação Acaraú Terra Sol no agropolo do Baixo Acaraú, Estado do Ceará. In: CARVALHO, E. B. S.; OLIVEIRA, J. L.; TROMPIERI NETO, N.; MEDEIROS, C. N.; Sousa, F. J. (Org.) Economia do Ceará em Debate 2010. Fortaleza: IPECE, 2010. v. 1, p. 109-132.

XIN, Z.; AIKEN, R.; BURKE, J. Genetic diversity of transpiration efficiency in sorghum. Field Crops Research, v. 111, p. 74-80, 2009.

ZWIRTES, A. L.; CARLESSO, R.; PETRY, M. T.; KUNZ, J.; REIMANN, G. K. Desempenho produtivo e retorno econômico da cultura do sorgo submetida à irrigação deficitária. Engenharia Agrícola, v. 35, n. 4, p. 676-688, 2015.

Recebido em: 08/11/2017

Aceito em: 24/09/2018 